\title{
Ab Initio Nuclear Structure from Helium to Oxygen Final Report: 00-ERD-028
}

\author{
W.E. Ormand, P. Navratil
}

February 4, 2003

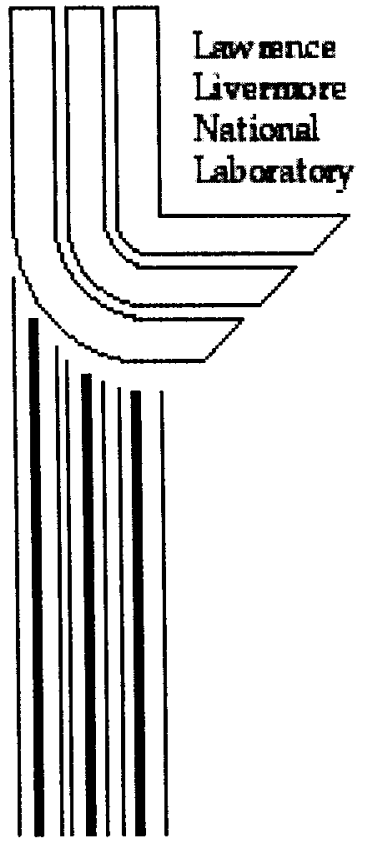




\section{DISCLAIMER}

This document was prepared as an account of work sponsored by an agency of the United States Government. Neither the United States Government nor the University of California nor any of their employees, makes any warranty, express or implied, or assumes any legal liability or responsibility for the accuracy, completeness, or usefulness of any information, apparatus, product, or process disclosed, or represents that its use would not infringe privately owned rights. Reference herein to any specific commercial product, process, or service by trade name, trademark, manufacturer, or otherwise, does not necessarily constitute or imply its endorsement, recommendation, or favoring by the United States Government or the University of California. The views and opinions of authors expressed herein do not necessarily state or reflect those of the United States Government or the University of California, and shall not be used for advertising or product endorsement purposes.

This work was performed under the auspices of the U. S. Department of Energy by the University of California, Lawrence Livermore National Laboratory under Contract No. W-7405-Eng-48.

This report has been reproduced directly from the best available copy.

Available electronically at http://www.doe.gov/bridge

Available for a processing fee to U.S. Department of Energy and its contractors in paper from

U.S. Department of Energy

Office of Scientific and Technical Information

P.O. Box 62

Oak Ridge, TN 37831-0062

Telephone: (865) 576-8401

Facsimile: (865) 576-5728

E-mail: reports@adonis.osti.gov

Available for the sale to the public from

U.S. Department of Commerce

National Technical Information Service

5285 Port Royal Road

Springfield, VA 22161

Telephone: (800) 553-6847

Facsimile: (703) 605-6900

E-mail: orders@ntis.fedworld.gov

Online ordering: http://www.ntis.gov/ordering.htm

OR

Lawrence Livermore National Laboratory Technical Information Department's Digital Library

http://www.llnl.gov/tid/Library.html 


\title{
$A b$ Initio Nuclear Structure from Helium to Oxygen Final Report: 00-ERD-028
}

\author{
W. Erich Ormand - PI \\ Petr Navratil - Co-PI
}

February 4, 2003

\begin{abstract}
Final report for a research program in theoretical nuclear physics to develop methods and computer programs to perform $a b$ initio calculations for light nuclei ranging from Helium to Oxygen. The method employed is based effective interaction theory within the framework of the shell model. The principal accomplishments were: 1) participation in the validation of $a b$ initio for ${ }^{4} \mathrm{He}$; the first study of $A=6$ nuclei with the realistic, non-local CD-Bonn potential; 3) a study of "intruder" states in $\left.{ }^{8} \mathrm{Be}, 4\right)$ the first shell-model calculations implementing three-body clusters in the effective interaction for nuclei with $A>4$; 5) the discovery that two-nucleon interactions by themselves are inadequate to describe the structure of the nucleus ${ }^{10} \mathrm{~B}$, which provides proof that three-nucleon forces affect nuclear structure; 6 ) first calculations including the Tucson-Melbourne three-nucleon force, which gives better agreement with the experimental spectrum for ${ }^{10} \mathrm{~B}$; and 7) the development of a new shellmodel code designed for public release.
\end{abstract}

\section{Introduction}

The study of how nuclei are put together is of fundamental interest to physics and the application of nuclear physics to National Security. The mechanics behind the structure of nuclei is an intellectually challenging enterprise, and plays a significant role in our understanding of essentially everything from quarks to cosmology. Nuclei are important laboratories where we study the smallest constituents of matter and the fundamental interactions and symmetries of nature. Further, nuclear reactions power the stars and forged the elements during the Big Bang and supernova explosions. Because of this ubiquitous presence, an understanding of nuclear structure is essential.

This document outlines work performed under an LDRD-supported research program $(00-$ ERD-028) in nuclear structure. The primary goal was to exploit recent advances in manybody theory and computational abilities to provide detailed descriptions of the structure of light nuclei from first principles. To accomplish this, a series of computer programs based on effective-interaction theory and utilizing the ASCI facility were developed to provide realistic models for nuclei ranging from ${ }^{4} \mathrm{He}$ to ${ }^{16} \mathrm{O}$. The primary accomplishments during the course of the project were: 1) participation in the validation of $a b$ initio for ${ }^{4} \mathrm{He}$; the first study of $A=6$ nuclei with the realistic, non-local CD-Bonn potential; 3) a study of "intruder" states in $\left.{ }^{8} \mathrm{Be}, 4\right)$ the first shell-model calculations implementing three-body clusters in the effective interaction for nuclei with $A>4 ; 5$ ) the discovery that two-nucleon interactions by themselves are inadequate to describe the structure of the nucleus ${ }^{10} \mathrm{~B}$, which provides proof that threenucleon forces affect nuclear structure; 6) first calculations including the Tucson-Melbourne three-nucleon force, which gives better agreement with the experimental spectrum for ${ }^{10} \mathrm{~B}$; and 
7) the development of a new shell-model code designed for public release. Overall, our work has been well received within the nuclear physics community as a significant development towards a fundamental description of the structure of atomic nuclei. This is highlighted by several publications in Physical Review Letters (the premier journal in physics) and several invited talks to conferences and university seminars and colloquia. Furthermore, our work has laid the foundation for new theory for nuclear reactions; also based on the fundamental interactions between nuclei. We are now developing this formalism, which will likely lead to a considerable enhancement in our capability to accurately predict reaction probabilities for thermonuclear reactions, which are important for both stellar astrophysics and Lab programs.

\section{Effective interactions and the shell model}

An important goal in the study of nuclear structure is to answer the question: Do we really understand how nuclei are put together? Towards this end, we wish to formulate a complete description of the properties of complex nuclei from first principles. In particular, we wish to determine if our knowledge of the fundamental interaction between pairs of nucleons is sufficient to describe the rich and complex structure observed in nuclei. This is an extremely difficult enterprise, and has really only been accomplished for the lightest of nuclei. Thus far, Faddeev-like [1, 2] approaches and the hyper-spherical formalism [3, 4] have been applied to three- and four-body systems, while Monte Carlo methods $[5,6]$ have now been applied to systems with up to ten nucleons [7]. Also, the coupled-cluster expansion method has been applied to ${ }^{16} \mathrm{O}[8]$. In this project, we utilized new developments in many-body theory and the exceptional computational power of the ASCI system to perform a study of nuclear structure from first principles, i.e., an $a b$ initio approach, for nuclei throughout the $p$-shell. Our primary focus has been to develop new capabilities, such as including three-body clusters into the effective interaction, and carry out a systematic study of nuclear structure using fundamental interactions.

The basic task at hand is to obtain solutions to the standard eigenvalue problem

$$
\left(\hat{H}-E_{\nu}\right) \Psi_{\nu}=0,
$$

where $E_{\nu}$ is the desired eigenvalue, $\hat{H}$ is the Hamiltonian, and $\Psi_{\nu}$ is the eigenfunction. The Hamiltonian is a key ingredient as it describes the kinetic motion of the constituent particles and their interaction with one another. Of particular importance is the interaction, which has been studied experimentally and predominantly acts between pairs of nucleons.

One starting point for solving Eq. (1) is the interacting shell-model [9]. The foundation of the shell model is the introduction of a set of orthogonal basis states $\phi_{i}$ that will be used to construct the exact solution, i.e., $\Psi_{\nu}=\sum_{i} c_{\nu i} \phi_{i}$. Solutions to Eq. (1) can then be obtained from a set of coupled equations that can be solved using matrix diagonalization techniques. The primary difficulty encountered with shell-model techniques is that because of the shortrange repulsion in the nucleon-nucleon interaction, a proper description of nuclear structure essentially requires a basis of infinite dimension.

This infinite basis problem can, in principle, be circumvented by the use of effectiveinteraction theory. First, one chooses manageable subset of the original basis states, which is defined by the operator $\hat{P}$, leading to the slightly different eigenvalue problem

$$
\left(\hat{H}_{e f f}-E_{\nu}\right) \hat{P} \Psi_{\nu}=0
$$

where $\hat{P} \Psi_{\nu}$ is the projection of the exact solution onto the chosen model space, $E_{\nu}$ is again the eigenvalue, and $\hat{H}_{e f f}$ is an effective Hamiltonian that yields the exact solution of Eq. (1). The 
excluded space is then usually defined by the operator $\hat{Q}$, with $\hat{P}+\hat{Q}=1, \hat{P}^{2}=\hat{P}, \hat{Q}^{2}=\hat{Q}$, and $\hat{P} \hat{Q}=\hat{Q} \hat{P}=0$.

An important feature of $\hat{H}_{\text {eff }}$ is that it is composed of two-, three-, ..., n-body components even if the fundamental interaction is only pair-wise. The power of $H_{\text {eff }}$ is that it may provide a mechanism to carry out computationally tractable calculations while including the relevant physics. For most potentials, the dominant correlations in the effective interactions are at the two-body level, but for smaller $P$-space, the higher-body correlations are essential for a correct result.

There are two possible strategies to developing a suitable $\hat{H}_{\text {eff }}$. The first is the BlochHorowitz equation [10] and recently advocated by Haxton et al. [11] for many-body applications. The second approach, which we utilized, is based on a unitary transformation due to Lee and Suzuki [12]. This formalism is the foundation for the highly successful no-core shell model (NCSM) $[13,14]$. The procedure is based on finding the transformation, $e^{\omega}$, to the Hamiltonian so that the $P$ - and $Q$-spaces for the many-body problem are decoupled, i.e.,

$$
\hat{Q} e^{-\omega} \hat{H} e^{\omega} \hat{P}=0 .
$$

Strictly speaking, in this form, $\hat{H}_{\text {eff }}$ is not unitary, but can be made so. An $n$-particle cluster expansion for $\hat{H}_{\text {eff }}^{(n)}$ begins with the set of exact solutions $\left|k^{(n)}\right\rangle$ to the $n$-body problem. With the set of $n$-body states $\left|\alpha_{P}^{(n)}\right\rangle$ (with dimension $d_{P}$ ) and $\left|\alpha_{Q}^{(n)}\right\rangle$ used to define the $n$-body matrix elements of the effective Hamiltonian within the $P$ - and $Q$-spaces for the $A$-particle system, matrix elements of the operator $\omega$ are given by

$$
\begin{aligned}
\left\langle\alpha_{Q}^{(n)} \mid k^{(n)}\right\rangle & =\sum_{\alpha_{P}}\left\langle\alpha_{Q}^{(n)}|\omega| \alpha_{P}^{(n)}\right\rangle\left\langle\alpha_{P}^{(n)} \mid k^{(n)}\right\rangle \\
\left\langle\alpha_{Q}^{(n)}|\omega| \alpha_{P}^{(n)}\right\rangle & =\sum_{k}\left\langle\alpha_{Q}^{(n)} \mid k^{(n)}\right\rangle\left\langle\tilde{k}^{(n)} \mid \alpha_{P}^{(n)}\right\rangle
\end{aligned}
$$

where the tilde denotes the inverted matrix of $\left\langle\alpha_{P}^{(n)} \mid k^{(n)}\right\rangle$. Note that sum in Eq. (5) need only be carried out over any set of $d_{P}\left|k^{(n)}\right\rangle$ solutions with $\left\langle\alpha_{P}^{(n)} \mid k^{(n)}\right\rangle \neq 0$. The $n$-body cluster of the effective interaction is then determined from the hermitian effective Hamiltonian

$$
\hat{H}_{e f f}^{(n)}=\left[\hat{P}\left(1+\omega^{\dagger} \omega\right) P\right]^{-1 / 2}\left(\hat{P}+\hat{P} \omega^{\dagger} \hat{Q}\right) H(\hat{Q} \omega \hat{P}+\hat{P})\left[\hat{P}\left(1+\omega^{\dagger} \omega\right) P\right]^{-1 / 2} .
$$

Explicit formulae for the $n$-body matrix elements are given by Eqs. (9) and (10) in Ref. [14]. Three important features are evident with the Lee-Suzuki method. First, in the limit that $\hat{P} \rightarrow 1$, the effective interaction tends to the bare interaction. Second, it is not possible to expand $\hat{H}_{e f f}$ in terms of two-body, three-body, and progressively higher-body components in a perturbative manner. Third, $d_{P}$ exact solutions $\left|k^{(n)}\right\rangle$ are required to determine the $\hat{H}_{e f f}^{(n)}$. These exact solutions may be obtained by any method, e.g., large-basis shell-model calculations utilizing either the bare interaction or an $(n-1)$-body effective interaction, which then must projected onto the shell-model $\left|\alpha_{P}^{(n)}\right\rangle$ states.

For applications in the NCSM, the effective interactions are derived from large-basis shellmodel calculations for the $n$-body system. In order to extend the model space to the largest possible extent these calculations were performed using a translationally-invariant harmonic oscillator basis, i.e., using Jacobi coordinates. Within this basis, it is computationally feasible to carry out calculations for all the relevant spin and isospin channels for $n=2$ for over 200 shells, for $n=3$ up to 41 shells, while for $n=4$ up to $17-19$ shells. In general, we find that for most realistic $N N$-potentials, is possible to determine $\hat{H}_{\text {eff }}^{(2)}$ for essentially any many-body 
model space. The $n=3$ space provides sufficient convergence for all channels utilizing $\hat{H}_{e f f}^{(2)}$. For $n=4$, however, the computationally allowable space is sufficient only for the $J=0^{+}, T=0$ channel even with $\hat{H}_{e f f}^{(3)}$. Thus, to derive four-body effective interactions, we need to improve the convergence for the four-body problem itself. We also note that due to computational problems with building and antisymmeterizing the basis states, the translationally-invariant oscillator basis is not practical for more than six particles (currently, $A=6$ with $4 \hbar \Omega$ is the limit), and conventional shell-model techniques are more efficient for $A \geq 6$. The advantage of the Jacobi coordinates is that the intrinsic and center-of-mass degrees of freedom are separated exactly within the construction of the basis states, and consequently the total basis dimension is smaller. In addition, the effective interaction is naturally derived in terms of the relative coordinates of the $n$-body cluster. Consequently, the final step for $\hat{H}_{e f f}^{(n)}$ is a transformation from the intrinsic Jacobi coordinates to the $M$-scheme representation of the shell-model.

Our calculations begin with a two-body (also plus three-body) Hamiltonian for the $A$ nucleon system, which depends on the intrinsic coordinates alone. We utilize realistic interaction potentials that are derived from nucleon-nucleon scattering data. To facilitate our calculations, we introduce an $A$-nucleon harmonic-oscillator Hamiltonian acting only on the center-of-mass, whose effect is subtracted from the many-body calculation. The primary advantages of the harmonic oscillator are that it acts as pseudo mean field providing a convenient basis for expanding the many-body wave function and that the relative motion of the center-of-mass can be separated from the intrinsic degrees of freedom exactly. Within the harmonic-oscillator basis, we specify the $P$-space, designated by the maximum number, $N_{\max }$, of oscillator quanta, and construct the $A$-body basis. We then obtain the eigenvalues, $E_{\nu}$, using a shell-model code. This amounts to diagonalizing a symmetric matrix, whose dimensions are given by the number of $A$ body basis states. Although the dimensions can be quite large, efficient numerical techniques, such as Lanczos [15], exist that yield the lowest eigenvalues. The parameters governing our convergence are then: $N_{\max }$, defining the model-space; $n$, the number of clusters in the effective interaction; and $b=\sqrt{m \Omega / \hbar}$, the oscillator parameter setting the physical scale. Ideally, the NCSM solution is independent of these parameters. In practice, however, our best solution is obtained for the largest $N_{\max }$ that is computationally feasible and a value of the oscillator parameter where the binding energy is least sensitive.

Up until our effort at LLNL, the NCSM has been implemented using only $\hat{H}_{\text {eff }}^{(2)}$ for $A>4$. The strategy has been to study convergence while steadily increasing $N_{\max }[16,17,18,19]$. Overall, these studies validate the method and clearly indicate convergence for sufficiently large $N_{\max }$, albeit slowly in some cases, such as ${ }^{8} \mathrm{Be}$. There are several reasons for extending NCSM to higher-body clusters. First, for any $P$-space, effective interaction theory implies better overall convergence while including higher-body clusters. Given that the computational effort expands significantly with increasing $N_{\max }$ and/or $n$, a compromise between $N_{\max }$ and $n$ must be made. Hence, it is important to determine the rate of convergence in $n$ for a given $N_{\max }$. The second reason is that there is strong evidence for the existence of "true" three-body forces in nuclei. Since the techniques for handling the "true" and effective three-body interactions are identical, it is therefore, natural to include effective three-body interactions. A third reason is that by including higher-body clusters, we are increasing the overall correlations in our wave functions, and, hence, a better description of clustering effects might be attained. In particular, it is possible that a good description of the famous alpha-cluster states in ${ }^{12} \mathrm{C}$ and ${ }^{16} \mathrm{O}$ may only be possible by using $\hat{H}_{e f f}^{(4)}$.

As mentioned above, computational limitations impose a compromise in the choice of $N_{\max }$ and $\hat{H}_{e f f}^{(n)}$. This is due to the fact that for each increment in $N_{\max }$ (note that due to parity conservation, an increment is two units in $N_{\max }$ ) the number of $A$-body states increases dra- 
matically (roughly a factor of 20-50 for each increment). While for larger $n$, the number of interaction matrix elements increases and the sparsity of matrix decreases. In addition, the effective interaction itself is more difficult to evaluate for increasing $n$ and/or $N_{\max }$. Presently, the entire $p$-shell $(4 \leq A \leq 16)$ is computationally tractable for $N_{\max }=4$ and $n=3$, while for $A \leq 7, N_{\max }=6$ is possible. For $n=2$, calculations have been extended to $N_{\max }=10$ for $A \leq 8[17,18]$ and it appears that $N_{\max }=10$ may be tractable for $A \geq 9[19,20]$. To illustrate the level complexity of the three-body calculations, for $N_{\max }=4,39,523,066$ 3-particle interaction matrix elements are needed. In this space, the number of $M$-scheme 10-body configurations for ${ }^{10} \mathrm{~B}$ with $J_{z}^{\pi}=0^{+}$is 581,740 , and the resultant matrix to be diagonalized has over $2.2 \times 10^{9}$ non-zero elements. Presently, we require approximately 150-200 CPU-node hours to obtain the lowest ten states for this system.

It should be pointed out that our project is complementary to $a b$ initio efforts utilizing the Green's Function Monte Carlo (GFMC) technique [5]. In particular, the GFMC and the $a b$ initio shell model provide independent checks on quality of their results. At present, the NCSM also enjoys some advantages over the GFMC method in terms of applicability. The first is that the NCSM is capable of utilizing non-local potentials, such as the effective-field theory (EFT) potentials derived from Chiral Lagrangians. Consequently, it is well suited to test the effectiveness of these new and promising formulations of inter-nucleon interactions. The second is that for the time being at least, the NCSM is computationally easier to extend to nuclei with

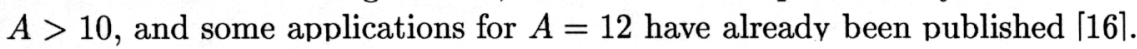

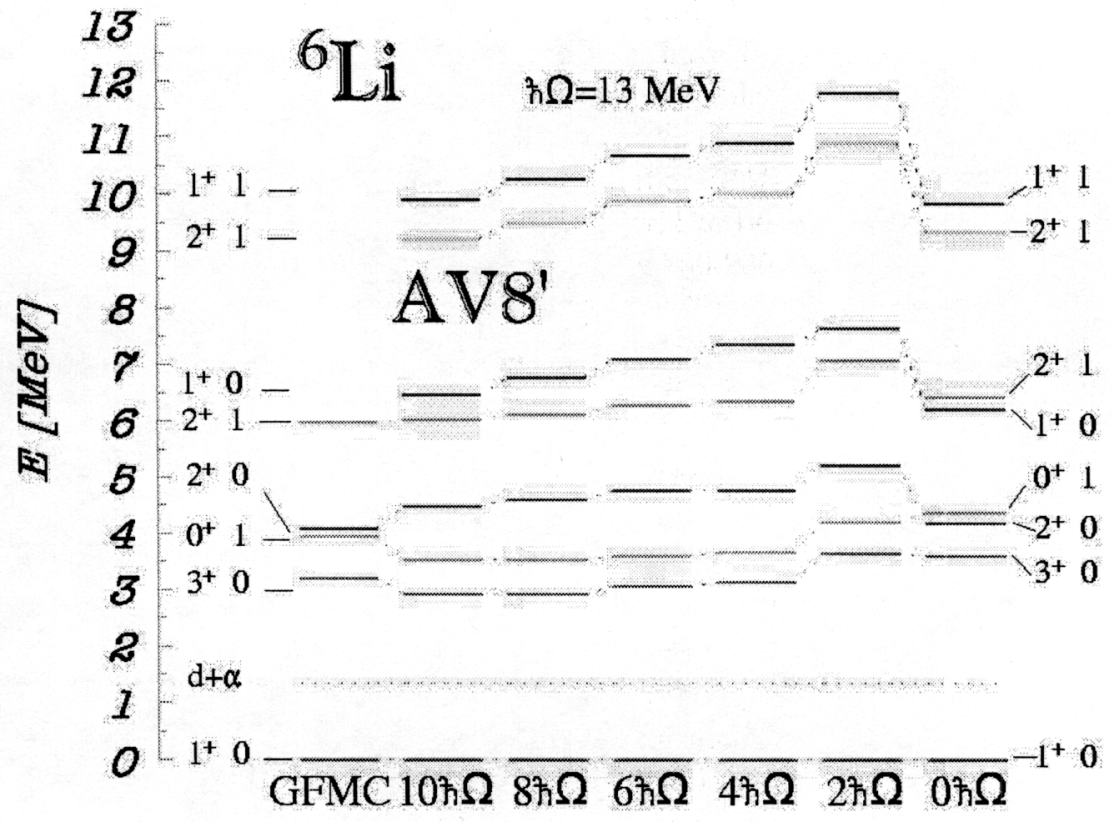

Figure 1: Comparison of the NCSM and GFMC spectra obtained for the Argonne AV8' potential. The NCSM spectra are shown as a function of the model size denoted by $N_{\max } \hbar \Omega$. 


\section{Accomplishments}

Over course of this project, we have developed new tools, which we outline in Subsections 3.2 and 3.3 below. With these tools, we then pursued our main objective to study the structure of light nuclei using realistic inter-nucleon forces. The results of these studies are outlined in Subsection 3.1
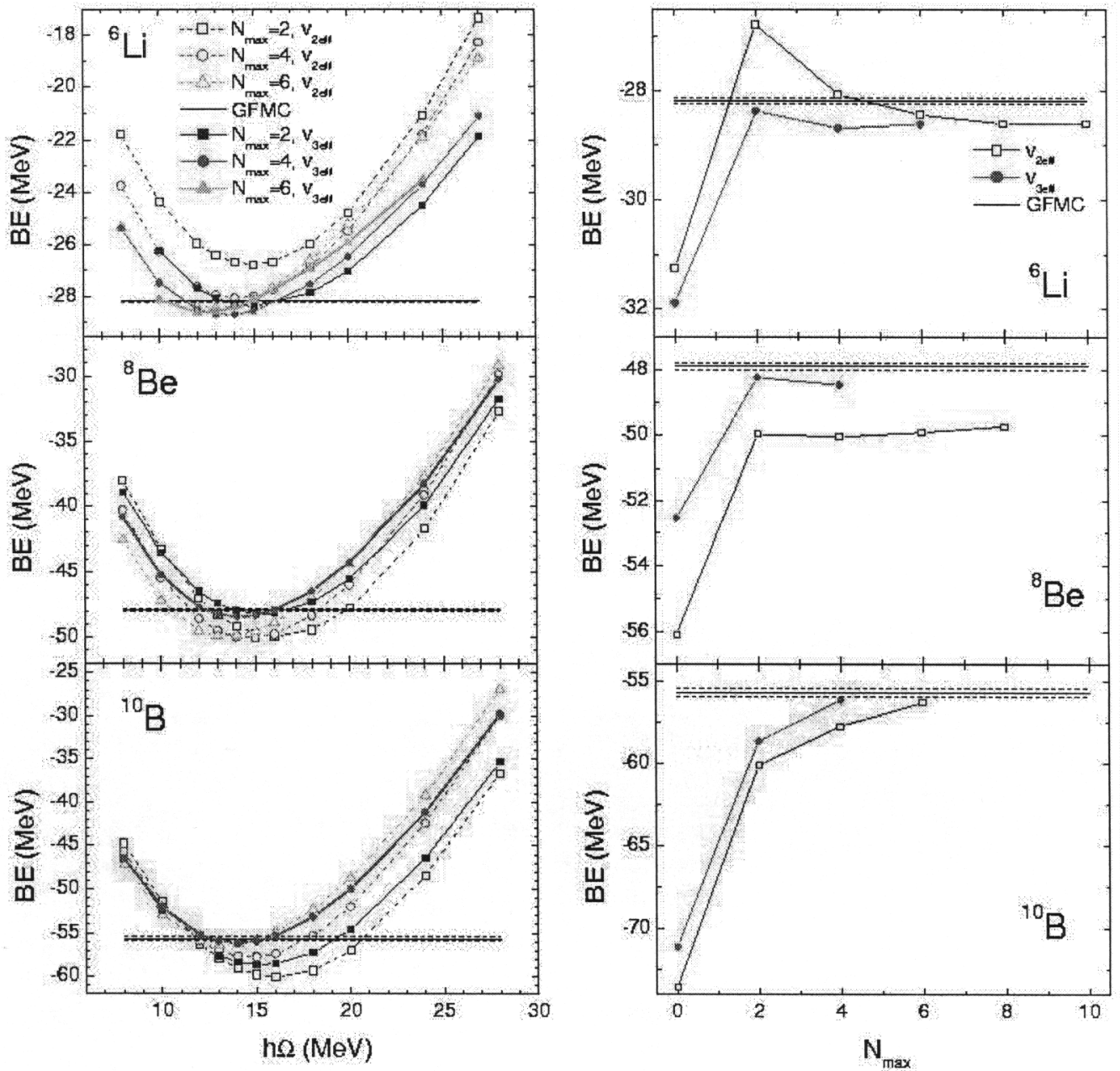

Figure 2: Calculated ground-state energy of ${ }^{6} \mathrm{Li}$ (upper panel), ${ }^{8} \mathrm{Be}$ (middle panel) and ${ }^{10} \mathrm{~B}$ (lower panel) using the AV8 ${ }^{\prime}$ NN potential with Coulomb. Results using the two-body effective interaction and the three-body effective interaction in basis spaces up to $6 \hbar \Omega$ in the range of $\mathrm{HO}$ frequencies of $\hbar \Omega=8-28 \mathrm{MeV}$ are shown and compared to the GFMC results from Ref. [6]. On the rhs, the energies at the HO frequency minima as a function of $N_{\max }$ are plotted.

\subsection{Nuclear Structure Calculations}

A research effort in $a b$ initio approaches to nuclear structure has been carried out with LDRD support at LLNL for the past three years. A fairly extensive list of our accomplishments is: 
1. Participation in a validation of $a b$ initio methods to ${ }^{4} \mathrm{He}$ (here, the effects of $\hat{H}_{e f f}^{(3)}$ were included) [21].

2. Study of ${ }^{6} \mathrm{Li}$ and the existence of soft-dipole modes in ${ }^{6} \mathrm{He}$ [17].

3. Study of inelastic proton scattering on ${ }^{6} \mathrm{Li}$ and ${ }^{6} \mathrm{He}[22]$.

4. Study of "intruder" states in ${ }^{8} \mathrm{Be}[18]$.

5. Calculations for $A=10$ nuclei, with an emphasis on isospin-mixing corrections to the superallowed Fermi beta decay [19].

6. Development of generalized computer programs to evaluate three-body effective interactions for shell-model calculations (manuscript under preparation).

7. First implementation of three-body effective interactions for systems with $A>4$ [23].

8. Discovery that two-nucleon interactions by themselves are inadequate to describe the structure of the nucleus ${ }^{10} \mathrm{~B}$, which provides proof that three-nucleon forces affect nuclear structure [23].

9. First calculations including the Tucson-Melbourne three-nucleon force, which gives better agreement with the experimental spectrum for ${ }^{10} \mathrm{~B}$ (manuscript under preparation).

10. Development of a new shell-model code (REDSTICK) designed for public release.

The study with $A=6$ provides an excellent example of the convergence and the utility of the no-core shell model. In particular, in Fig. 1, we compare the NCSM spectrum for ${ }^{6} \mathrm{Li}$ (as a function of the model space $N_{\text {max }}$ ) using the Argonne AV8' potential with results obtained from the Green's Function Monte Carlo method. Overall, there is good agreement between the two methods. Also, as will be shown below, the converged NCSM value for the total binding energy ${ }^{6} \mathrm{Li}$ is agreement to within $400 \mathrm{keV}$ of the GFMC calculation.

The inclusion of higher-body clusters generally improves the overall convergence. This was the object of the study published in Ref. [23]. The Binding energies of the nuclei ${ }^{6} \mathrm{Li},{ }^{8} \mathrm{Be}$, and ${ }^{10} \mathrm{~B}$ are shown in Fig. 2. On the left-side of the figure the binding energies are plotted as a function of the oscillator parameter (in this figure denoted by $\hbar \Omega$, which effectively defines the size of the nucleus. The figure shows parabolas for the various model spaces, which are denoted in the upper left-hand corner by the $N_{\text {max }}$ value, and with two-body ( $V_{2 e f f}$ - dotted lines) and three-body ( $V_{3 e f f}$ - solid lines) effective interactions. In general, the behavior on the oscillator parameter is lessened (flatter parabola) as either the model space size increases or when more clusters are included in the effective interaction. As mentioned above, the "best" result for a given model space is chosen in the region exhibiting the least dependence on the oscillator parameter. These "best" values are then plotted on the right-side of the figure as a function of the model space $N_{\max }$ and compared with the results from the GFMC method (full solid lines with a dotted line band denoting the GFM uncertainty). In general, for any given value of $N_{\max }$, faster convergence is achieved with higher clusters included in $H_{\text {eff }}$. In addition, we note that the NCSM calculation with the two-body effective interaction still differs from the GFMC result by $\approx 1.8 \mathrm{MeV}$ even for the largest model space. On the other hand, the three-body effective interaction results are in better agreement for smaller model spaces. Given that ${ }^{8} \mathrm{Be}$ is actually unbound, and is two alpha-particle resonance, this suggests that the three-body effective interactions includes more correlations into the wave function. Overall, the results obtained with the three-body clusters in the effective interaction are in agreement with the GFMC calculations to within $400 \mathrm{keV}$. 


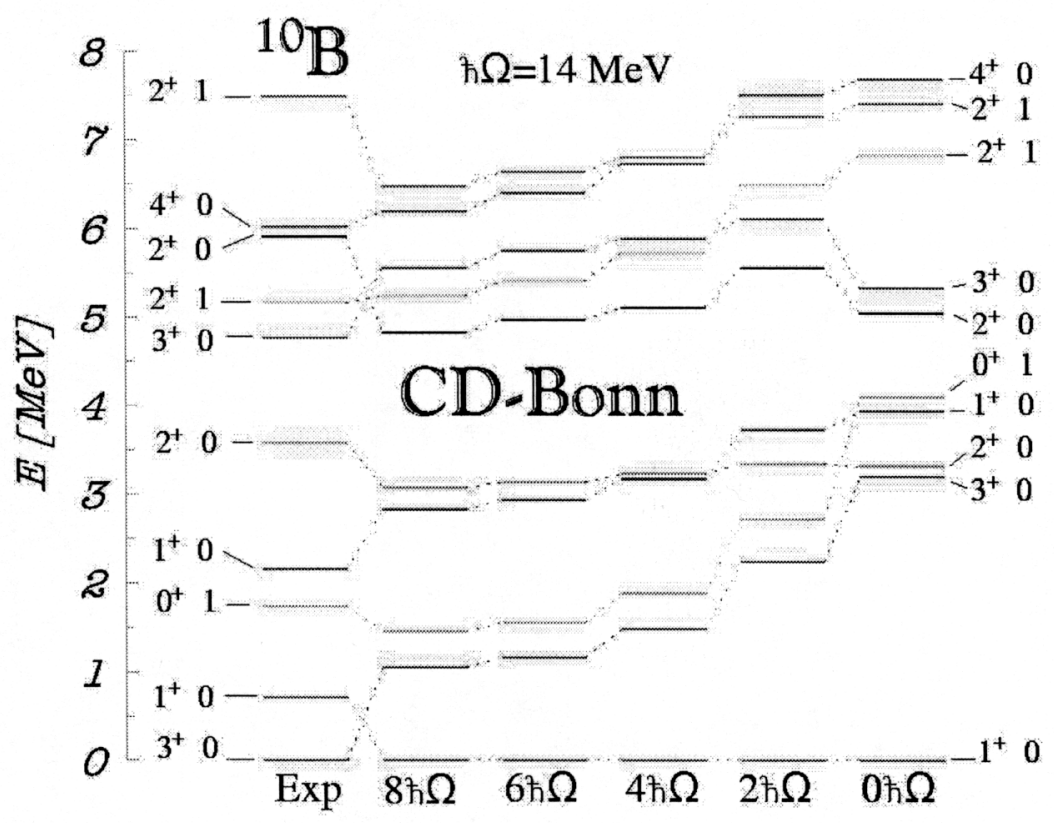

Figure 3: The low-lying spectrum of ${ }^{10} \mathrm{~B}$ as a function of the model space size $N_{\max }$ compared to experiment.

With confidence in convergence, we now turn to a more systematic study of the structure of light nuclei. A particularly salient example is ${ }^{10} \mathrm{~B}$, which we show in Fig. 3. Here, we compare the NCSM spectra as a function of $N_{\max }$ with that observed experimentally. The most striking feature is that the ground state $\left(3^{+}\right)$and the first excited state $\left(1^{+}\right)$are reversed in order. We must now conclude that realistic two-nucleon forces fail to describe the low-lying structure of ${ }^{10} \mathrm{~B}$. Indeed, this is a feature that appears to be common to all the realistic nucleon-nucleon forces, and in particular, CD-Bonn [24] and the Argonne [6]. This is the first direct evidence that, in addition to providing extra binding, three-nucleon forces also impact nuclear structure.

At this point, we are forced to include that a proper description of nuclear structure must a include so-called "true" three-nucleon force. Note that these are quite different from the threebody clusters that we included in the effective interaction, as these term are actually induced because of the effect of the finite model space. We have recently carried out a preliminary set of calculations including the Tucson-Melbourne three nucleon force [25] for ${ }^{10} \mathrm{~B}$. The results are shown in Fig. 4, where better agreement with the experimental spectrum is obtained, In particular, the ordering of the first two states is now correct. Overall, one finds that the threenucleon interactions has spin-orbit components that play an important role in determining the structure of nuclei in the region $10 \leq A \leq 15$. We should also note that even with the TucsonMelbourne force, there are still some disagreements with experiment, which indicates a need to fine tune its components.

\subsection{Tools for Effective Interactions}

The effective interaction requires several layers of processing. In general, more clusters put into the effective interaction leads to more extensive computation. The first step is obtaining 


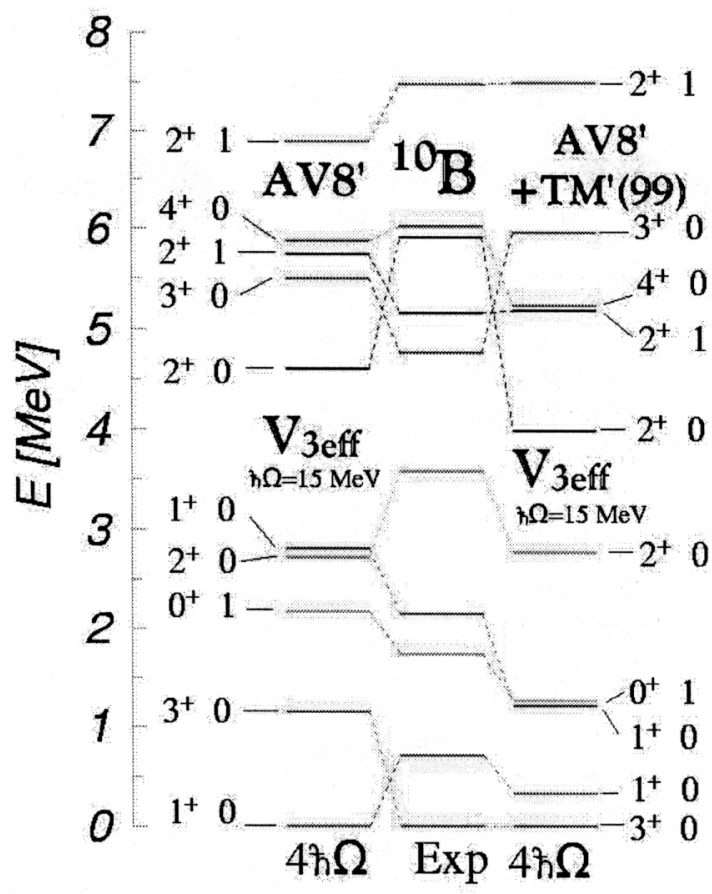

Figure 4: Comparison of low-lying spectrum of ${ }^{10} \mathrm{~B}$ obtained with the AV8' two-nucleon interaction alone(left side) and with the Tucson-Melbourne three-nucleon force (right side)with experiment.

converged $n$-body solutions. For a small number of particles, the utilization of a Jacobi coordinate basis permits a calculation to much larger $N_{\max }$. For this purpose, we rely exclusively on the Jacobi-basis program written by the Co-PI [14] while he was at the University of Arizona. With these solutions, we then perform the Lee-Suzuki transformation, and derive the effective interaction for the $A$-body system. The effective interaction is then transformed from the Jacobi coordinates into the $M$-scheme formalism suitable for large-basis shell-model calculations. At the two-body level, the transformation is fairly simple and depends on the classic BrodyMoshinsky brackets for harmonic oscillators. For the three-body case, we used an $M$-scheme three-body Slater-Determinant, harmonic-oscillator basis characterized by $M_{3}=m_{1}+m_{2}+m_{3}$, $M_{\mathrm{T} 3}=m_{\mathrm{t} 1}+m_{\mathrm{t} 2}+m_{\mathrm{t} 3}$ and parity, with $M_{3}$ and $M_{T 3}$ denoting the third component of the angular momentum and isospin, respectively. The transformation from the Jacobi basis is rather complex and depends on products of two harmonic-oscillator Brody-Moshinsky brackets (one for two particles with mass ratio 1 and a second for two particles with mass ratio $1 / 2$ ) and the parentage coefficients obtained by antisymmetrization of the Jacobi coordinate harmonicoscillator basis. In our work, these transformations were performed utilizing an computer program written by the co-PI.

Generally speaking, $H_{\text {eff }}^{(2)}$ is straightforward, and can be obtained for any model space within a matter of minutes. On the other hand, $H_{\text {eff }}^{(3)}$ is complicated and computationally expensive. Indeed, for many $A$-body applications, the CPU requirements to carry out the transformation are equal to (and often exceed) those for the $A$-body diagonalization. Furthermore, the calculation of the matrix elements of a bare true three-body interaction is even more time consuming, and often exceeds several days. 


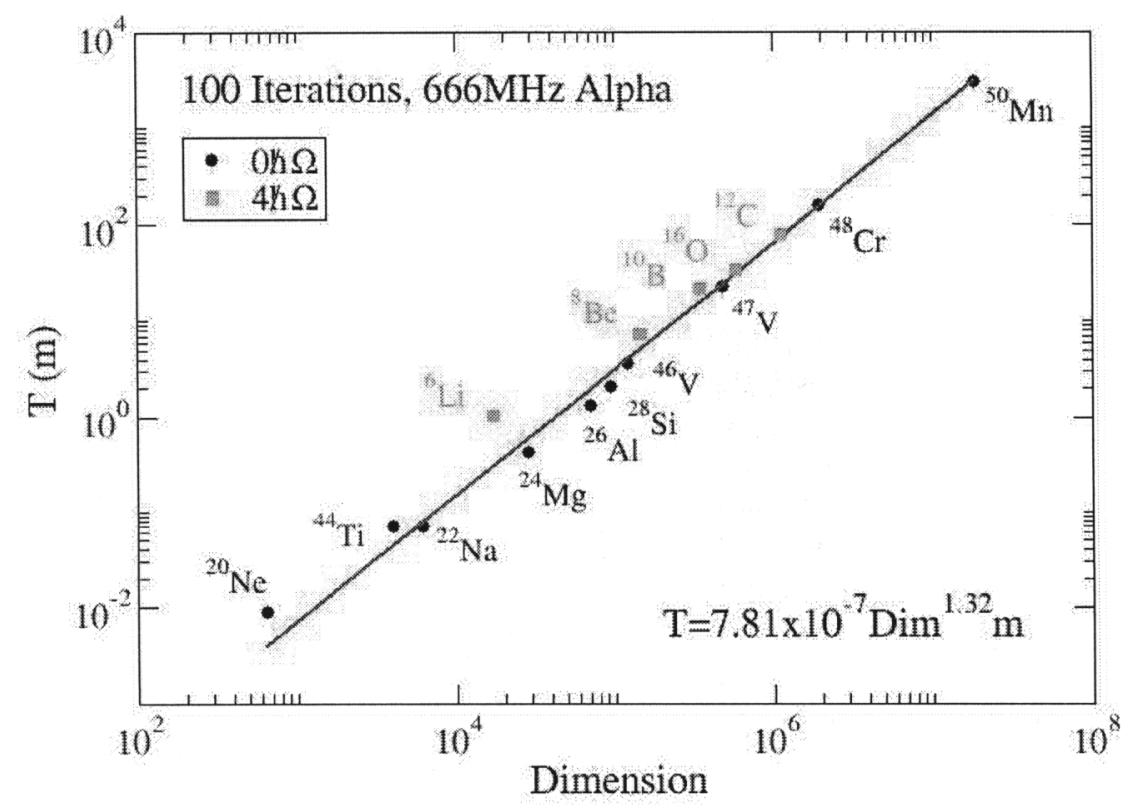

Figure 5: CPU time in minutes required by REDSTICK to perform 100 Lanczos iterations on a $666 \mathrm{MHz}$ alpha for various systems. The $p$-shell systems $\left({ }^{6} \mathrm{Li}\right.$ to $\left.{ }^{12} \mathrm{C}\right)$ were carried out for $N_{\max }=4(4 \hbar \Omega)$.

\subsection{Shell-Model Programs}

During the past three years, we have utilized several shell-model computer programs. These include the Many-Fermion Dynamics (MFD) code [26] and ANTOINE [27], a Jacobi coordinate based program written by the Co-PI [14], and the locally developed program REDSTICK primarily written by the PI. The general philosophy of REDSTICK is to use the Lanczos method to find a set of lowest eigenvalues. The Lanczos algorithm is applied to a basis vector "on-thefly". Meaning that the full Hamiltonian matrix is not stored on disk. Rather, information is stored internally that permits what amounts to an efficient calculation of the matrix for each iteration of the Lanczos procedure. For very large systems, this is tends to be more efficient as the input/output load is minimal. During the final year of the project, REDSTICK underwent a series of improvements that make it competitive with the best shell-model codes, such as ANTOINE [27] for systems with dimensions up to 50,000,000. It is to be noted that REDSTICK was designed for a public distribution, and in many ways is better documented and easier to use.

The serial performance for REDSTICK as a function of the model space is shown in Fig. 5, where a nearly linear behavior is obtained as a function of the basis size. The program was also written to work in serial and parallel modes (with MPI) and is flexible enough to handle large $\hbar \Omega$ calculations as well as standard shell-model applications. It has been applied to typical full-space $f p$-shell nuclei such as ${ }^{50} \mathrm{Mn}$ as well as $\mathrm{Sn}$ and In isotopes, where the protons and neutrons occupy different model spaces. The plan is for this program to be reviewed and released for a full public distribution. The steps remaining prior to a public release of the main 
component of the program that will obtain wave functions and eigenenergies is the completion of documentation and some very small modifications to improve the efficiency in constructing of basis states for systems with a large number of particles. An auxiliary program supporting the main code by computing spectroscopic factors, and one- and two-body densities is under development and will also be reviewed and released for public distribution. Once released, the current code is powerful new tool for the nuclear physics community. Efforts are currently underway to implement further improvements that can extend the applicability of the program to problems with several hundred million basis states.

\section{Publications}

Over the course of the project, our work has been represented by publications in Physical Review journals and are listed below. These include two articles in the prestigious Physical Review Letters. Two other publications are currently under preparation. These include a detailed description of the method to calculate the three-body effective interaction and our recent results with three-nucleon interactions. In addition, the PI and co-PI have each presented in excess of 15 seminars, invited talks to conferences, and colloquia over the past two years.

List of publications in refereed journals:

1. H. Kamada, A. Nogga, W. Glöckle, E. Hiyama, M. Kamimura, K. Varga, Y. Suzuki, M. Viviani, A. Kievsky, S. Rosati, J. Carlson, S. C. Pieper, R. B. Wiringa, P. Navrátil, B. R. Barrett, N. Barnea, W. Leidemann and G. Orlandini, Benchmark test calculation of a four-nucleon bound state, Phys. Rev. C 64, 044001 (2001). Lawrence Livermore National Laboratory document UCRL-JC-143602.

2. E. Caurier, P. Navrátil, W. E. Ormand and J. P. Vary, Intruder states in ${ }^{8} \mathrm{Be}$, Phys. Rev. C 64, 051301 (2001). Lawrence Livermore National Laboratory document UCRL-JC144859 .

3. P. Navrátil, J. P. Vary, W. E. Ormand and B. R. Barrett, Six nucleon spectroscopy from realistic non-local Hamiltonian, Phys. Rev. Lett. 87, 172502 (2001). Lawrence Livermore National Laboratory document UCRL-JC-143670.

4. P. Navrátil and W. E. Ormand, $A b$ initio shell model calculations with three-body effective interactions for $p$-shell nuclei. Phys. Rev. Lett. 88, 152502 (2002). Lawrence Livermore National Laboratory document UCRL-JC-146446.

5. E. Caurier, P. Navrátil, W. E. Ormand and J. P. Vary, $A b$ initio shell model for $A=10$ nuclei, Phys. Rev. C 66, 024314 (2002). Lawrence Livermore National Laboratory document UCRL-JC-148460.

6. D. C. J. Marsden, P. Navrátil, S. A. Coon and B. R. Barrett, Feasibility study of a threenucleon force in the no-core shell model: ${ }^{3} \mathrm{H}$ binding energy. Phys. Rev. C 66, 044007 (2002). Lawrence Livermore National Laboratory document UCRL-JC-148973.

\section{University Partnerships}

The research presented in this proposal involved collaborations with and scientists in the academic community; in particular: W.C. Haxton (University of Washington), B.R. Barrett (University of Arizona), J.P. Vary (Iowa State University), C.W. Johnson (San Diego State University and San Diego State University), and E. Caurier (CNRS - Strasbourg). 


\section{The Future}

In many ways, the future for nuclear structure is bright, and we are entering exciting times. This is evidenced by increased interest nationwide in attempts to describe light nuclei from fundamental interactions. As we pointed out earlier, past descriptions of nuclear structure were largely hampered by insufficient computation capability, and effective interactions were determined empirically, instead of from a fundamental theory. Now, with vast improvements in computer technology, many problems in nuclear physics that were once intractable, are now beginning to be feasible.

We plan to continue this project, and are even expanding it into a completely new direction. Presently, we are attempting to use our success during the past three years into support for a post doctoral fellow from the DOE Office of Science to further our investigations. Our primary goals are:

1. Carry out an extensive study of the form of the "true" three-nucleon force to understand its form.

2. Extend our capability to include four-body clusters in the effective interactions.

3. Explore the viability of a new class of inter-nucleon potentials, referred to as effective field theory (EFT) potentials. These EFT potentials are based on fundamental properties of quantum chromo-dynamics (the underlying theory of the strong nuclear force) and show considerable promise. Since these potentials are non-local, ours is essentially method that can perform this investigation.

4. Study improvements to the "mean-field" used in the calculations. Although the oscillator basis used here is convenient and is really the only one in which an exact separation between the intrinsic and center-of-mass motions can be made, it does a poor job of describing the radial behavior of the wave functions at large distances. We plan a study using another basis to see if the method can be improved.

5. To implement effective operators for transitions. Just as the Hamiltonian must be renormalized into and effective interaction, the same is true for all other operators, including those governing transitions. For now, the primary focus has been on the development of the tools to treat the effective interaction.

6. Develop a mechanism to describe low-energy fusion reactions between light ions. In this case, the NCSM is used to first describe the independent intrinsic structures of the target and projectile nuclei and the composite system. A dynamic motion of the target and projectile using the full Hamiltonian and the overlap between the composite system and the target and projectile as a function of their separation is then solved. This would then provide the capability to accurately calculate reaction cross sections for thermonuclear reactions, which are important for stellar astrophysics and Lab programs.

\section{Acknowledgements}

This work was performed under the auspices of the U.S. Department of Energy by the University of California, Lawrence Livermore National Laboratory, under contract No. W-7405-Eng-48. This project received support through a Laboratory Directed Research and Development grant, tracking No. 00-ERD-028. 


\section{References}

[1] L.D. Faddeev, Zh. Éksp. Teor. Fiz. 39, 1459 (1960) [Sov. Phys. JETP 12, 1014 (1961)]; O.A. Yakubovsky, Yad. Fiz. 5, 1312 (1966) [Sov. J. Nucl. Phys. 5, 937 (1967)].

[2] H. Kamada and W. Glöckle, Nucl. Phys. A548, 205 (1992); J.L. Friar, G.L. Payne, V.G.J. Stoks, and J.J. de Swart, Phys. Lett. B 311, 4 (1993); H. Witala, D. Hüber, and W. Glökle, Phys. Rev. C49, R14 (1994); A. Nogga, D. Hüber, H. Kamada, and W. Glökle, Phys. Lett. B 409, 19 (1997); W. Glökle and H. Kamada, Phys. Rev. Lett. 71, 971 (1993).

[3] M. Viviani, A. Kievsky, and S. Rosati, Few-Body Syst. 18, 25 (1995).

[4] N. Barnea, W. Leidermann, and G. Orlandini, Nucl. Phys. A650, 427 (1999).

[5] J. Carlson, Phys. Rev. C36, 2026 (1987); C38, 1879 (1988); J. Carlson and R. Schiavilla, in Few-Body Systems, suppl. 7, Ed. by B.L.G. Bakker and R. van Dantzig (Springer-Verlag, 1994) p. 349.

[6] B.S. Pudliner et al, Phys. Rev C56, 1720 (1997); R.B. Wiringa, Nucl. Phys. A631, 70c (1998); R.B. Wiringa, S.C. Pieper, J. Carlson, and V.R. Pandharipande, Phys. Rev. C62, 014001 (2000); S.C. Pieper, V.R. Pandharipande, R.B. Wiringa, and J. Carlson, Phys. Rev. C64, 014001 (2001).

[7] S.C. Pieper, private communication.

[8] J.H. Heisenberg and B. Mihaila, Phys. Rev. C59, 1440 (1999); B. Mihaila and J.H. Heisenberg, Phys. Rev. C61, 054309 (2000); B. Mihaila and J.H. Heisenberg, Phys. Rev. Lett. 84, 1403 (2000).

[9] P.J. Brussaard and P.W.M. Glaudemans, Shell-model applications in nuclear spectroscopy, (North-Holland, Amsterdam, 1977); R.D. Lawson, Theory of the nuclear shell model, (Clarendon Press, Oxford, 1980).

[10] C. Bloch and J. Horowitz, Nucl. Phys. 8, 91 (1958).

[11] W.C. Haxton and C.-L. Song, Phys. Rev. Lett. 84, 5484; W.C. Haxton and T. Luu, Nucl. Phys. A690, 15 (2001)

[12] K. Suzuki and S.Y. Lee, Prog. Theor. Phys. 64, 2091 (1980); K. Suzuki, Prog. Theor. Phys. 68, 246 (1982).

[13] P. Navrátil and B.R. Barrett, Phys. Rev. C57, 562 (1998); P. Navrátil and B.R. Barrett, Phys. Rev. C59, 1906 (1999).

[14] P. Navrátil, G.P. Kamuntavičius, and B.R. Barrett, Phys. Rev. C 61, 044001 (1998).

[15] J.H. Wilkinson, The Algebraic Eigenvalue Problem, (Clarendon Press, Oxford, 1965).

[16] P. Navrátil, J.P. Vary, and B.R. Barrett, Phys. Rev. Lett. 84, 5728 (2000); P. Navrátil, J.P. Vary, and B.R. Barrett, Phys. Rev. C62, 054311 (2000).

[17] P. Navrátil, J.P. Vary, W.E. Ormand, and B.R. Barrett, Phys. Rev. Lett. 87, 172501 (2001).

[18] E. Caurier, P. Navrátil, W.E. Ormand, and J.P. Vary, Phys. Rev. C64, 051301 (2001) 
[19] E. Caurier, P. Navrátil, W.E. Ormand, and J.P. Vary, Phys. Rev. C66, 024314 (2002).

[20] E. Caurier (private communication).

[21] H. Kamada et al., Phys. Rev. C64, 044001 (2001).

[22] V. Lapoux et al., (in press).

[23] P. Navrátil and W.E. Ormand Phys. Rev. Lett. 88, 152502 (2002)

[24] R. Machleidt, F. Sammarruca and Y. Song, Phys. Rev. C53, 1483 (1996).

[25] S.A. Coon, M.D. Scadron, P.C. Mcname, B.R. Barrett, D.W.E. Blatt, B.M.J. McKellar, Nucl. Phys. A317, 242 (1979).

[26] J.P. Vary, "The Many-Fermion-Dynamics Shell-Model Code", Iowa State University (1992) (unpublished); J.P. Vary and D.C. Zheng, ibid., (1994).

[27] E. Caurier et al, Phys. Rev. C59, 2033 (1999); E. Caurier and F. Nowacki, Acta Physica Polonica 30, 705 (1999). 